\title{
Temporal Trends in Clinical Outcomes Following Percutaneous Coronary Intervention in Patients with Renal Insufficiency
}

\author{
Ryo Naito, Katsumi Miyauchi, Jun Shitara, Hirohisa Endo, Hideki Wada, Shinichiro Doi, Hirokazu Konishi, \\ Shuta Tsuboi, Manabu Ogita, Tomotaka Dohi, Takatoshi Kasai, Hiroshi Tamura, Shinya Okazaki, \\ Kikuo Isoda and Hiroyuki Daida
}

Department of Cardiovascular Medicine, Juntendo University Graduate School of Medicine, Tokyo, Japan

\begin{abstract}
Aim: Renal insufficiency is associated with worse clinical outcomes in patients with coronary artery disease. Since the introduction of percutaneous coronary intervention (PCI), the revascularization therapy has evolved with advances of devices, improvements in operator techniques, and the establishment of medical therapy. We examined temporal trends of the clinical outcomes following PCI in patients with renal insufficiency.

Methods: Patients with renal insufficiency after PCI at Juntendo University across three eras (plain balloon angioplasty, bare metal stent (BMS), and drug-eluting stent (DES)) were examined in this study. The primary endpoint was a composite of all-cause mortality, nonfatal acute coronary syndrome, nonfatal stroke, and repeat revascularization within 3-years after the index revascularization. Results: A total of 1,420 patients were examined. Baseline characteristics have become unfavorable over time, whereas administration rate of medications for secondary prevention has increased. The event-free survival rates for the endpoint were different among the groups. Adjusted relative risk reduction for the endpoint was 35\% and 51\% in the BMS and DES eras (using the plain angioplasty era as reference). The adjusted relative risk reduction of the DES era was $26 \%$ compared with that of the BMS era.

Conclusions: The incidence of cardiovascular events after PCI has reduced during the 26-year period mainly because of the reduction in repeat revascularization in patients with renal insufficiency, despite the higher risk profiles in the recent era.
\end{abstract}

Key words: Renal insufficiency, Percutaneous coronary intervention, Temporal trends

\section{Introduction}

Renal insufficiency is an independent risk factor for coronary artery disease (CAD) ${ }^{1-6)}$. Furthermore, previous studies reported that CAD patients with renal insufficiency had worse clinical outcomes compared with those without renal insufficiency ${ }^{7-9)}$. Since the introduction of plain old balloon angioplasty (POBA) for CAD in 1977, percutaneous coronary intervention (PCI) has evolved because of the technological advances of devices, improvements in operator

Address for correspondence: Katsumi Miyauchi, Department of Cardiovascular Medicine, Juntendo University Graduate School of Medicine, 2-1-1 Hongo Bunkyo-ku, Tokyo 1138421, Japan

E-mail: ktmmy@juntendo.ac.jp

Received: December 7, 2015

Accepted for publication: January 5, 2016 techniques, and the establishment of medical therapy for secondary prevention ${ }^{10-12)}$. In particular, the advent of drug-eluting stents (DESs) has significantly reduced the occurrence of target lesion revascularization from $20 \%$ to $30 \%$ in the bare metal stent (BMS) era to below $\left.10 \%{ }^{13}, 14\right)$. Recently, we reported that clinical outcomes following PCI improved across POBA, BMS, and DES era ${ }^{15)}$. Therefore, it is of interest to know the temporal trends of clinical outcomes after PCI in patients with renal insufficiency who were at high risk of morbidity and mortality.

\section{Aim}

The aim of this study was to know the temporal trends of clinical outcomes following PCI in CAD patients with renal insufficiency. 


\section{Methods}

\section{Study Population}

We analyzed the data from database of the Juntendo PCI Registry in which information of patients who underwent PCI at Juntendo University Hospital (Tokyo, Japan) from January 1984 to February 2010 was available. This database has been maintained since the introduction of PCI in our institution. The study population was divided into three groups according to the time period of the index PCI (POBA era: January 1984-December 1997, BMS era: January 1998-July 2004, and DES era: August 2004-February 2010).

\section{Clinical Outcomes}

The endpoints included major adverse cardiovascular events that occurred within 3 years after the index PCI procedure. MACE was defined as one or more of the following: (1) all-cause mortality, (2) nonfatal acute coronary syndrome (ACS), (3) nonfatal stroke, and (4) repeat revascularization. Information regarding the clinical outcomes was collected during clinical visits, via telephone interviews or from the referring physician. The Institutional Review Board of Juntendo University Hospital approved the protocol of this study, which was performed in accordance with the principles established in the Declaration of Helsinki and our institutional ethics policy, and written informed consent was obtained from all study participants.

\section{Definitions}

Renal insufficiency was defined as an estimated glomerular filtration rate (eGFR) of $<60 \mathrm{ml} \cdot \mathrm{min}^{-1} \cdot 1.73$ $\mathrm{m}^{2}$, calculated using the modification of diet in renal disease (MDRD) equation modified with a Japanese coefficient using baseline serum creatinine ${ }^{16)}$. Diabetes mellitus was defined as glycated hemoglobin A1c (HbA1c) of National Glycohemoglobin Standardization Program (NGSP) value $\geq 6.5 \%$, by a previous diagnosis from medical records or being treated with any oral antidiabetic agents or insulin. We converted HbA1c [Japan Diabetes Society (JDS)] values to HbAlc (NGSP) units using the following equation: $\operatorname{NGSP}(\%)=1.02 \times \mathrm{JDS}(\%)+0.25 \%{ }^{17)}$. Hypertension was defined as systolic blood pressure $\geq 140 \mathrm{mmHg}$ and diastolic blood pressure $\geq 90 \mathrm{mmHg}$ or treatment with antihypertensive medications. Dyslipidemia was defined by a previous diagnosis from medical records, lipid profiles, i.e., triglyceride $\geq 150 \mathrm{mg} / \mathrm{dl}$, low-density lipoprotein cholesterol $\geq 140 \mathrm{mg} / \mathrm{dl}$ or high-density lipoprotein cholesterol $<40 \mathrm{mg} / \mathrm{dl}$ or being treated with antidyslipidemic medications. All these blood sampling data were measured in the morning of the day when the index PCI was performed, whereas in emergent cases, the measurement was performed at the timing of the emergent admission. We defined current smokers as individuals who smoked at the time of admission or who had quit within 1 year before the study period. ACS was defined as unstable angina pectoris (UAP), non-ST segment elevation myocardial infarction (NSTEMI) or STEMI. UAP was defined as angina at rest or in an accelerating pattern with negative cardiac biomarkers, with or without ECG changes indicative of myocardial ischemia (e.g., ST segment depression or transient elevation or new T-wave inversion). Myocardial infarction (MI) was diagnosed in the presence of two of the following three criteria: (1) typical chest pain for at least $20 \mathrm{~min}$, (2) increased serum creatine kinase level to twice the upper normal range, and (3) a new Q wave on electrocardiography. Procedural success of PCI was defined as a decrease in residual luminal diameter stenosis to $<50 \%$ or an achievement of thrombolysis in MI grade 3 in the final angiogram of the procedure.

\section{Statistical Analyses}

Continuous variables were expressed as the mean and standard deviation. Categorical data were expressed as counts and percentages. Continuous variables were compared using one-way analysis of variance followed by post-hoc analysis using Dunnett's test for multiple comparisons. The POBA era served as a control group in the post-hoc analysis. The continuous variables with statistical significance in the analysis are marked with *. Categorical variables were analyzed by the chi-square test or Fisher's exact probability test. Analysis of the intergroup comparison with the POBA era group for categorical variables was calculated with Bonferroni's correction. The categorical variables with statistical significance in the analysis are marked with ${ }^{\dagger}$. In the analysis, a $P$-value $<0.025$ was considered to be significant. Cochran-Armitage test for trend was performed to assess for presence of an association between the incidence of 3-year MACE and the PCI eras. Unadjusted cumulative event rates for 3-year MACE were estimated using Kaplan-Meier methods and compared using the log-rank test across the three groups. To identify whether the PCI eras (with the POBA era as the reference) were associated with the clinical outcomes following PCI, univariate Cox regression analysis was performed. Furthermore, multivariate Cox regression analysis, including age, gender, body mass index (BMI), hypertension, diabetes, dyslipidemia, current smoking, hemodialysis, and a presentation of ACS at the index PCI as confounding factors was performed. The hazard ratio (HR) and $95 \%$ confidence intervals were also calculated. A 


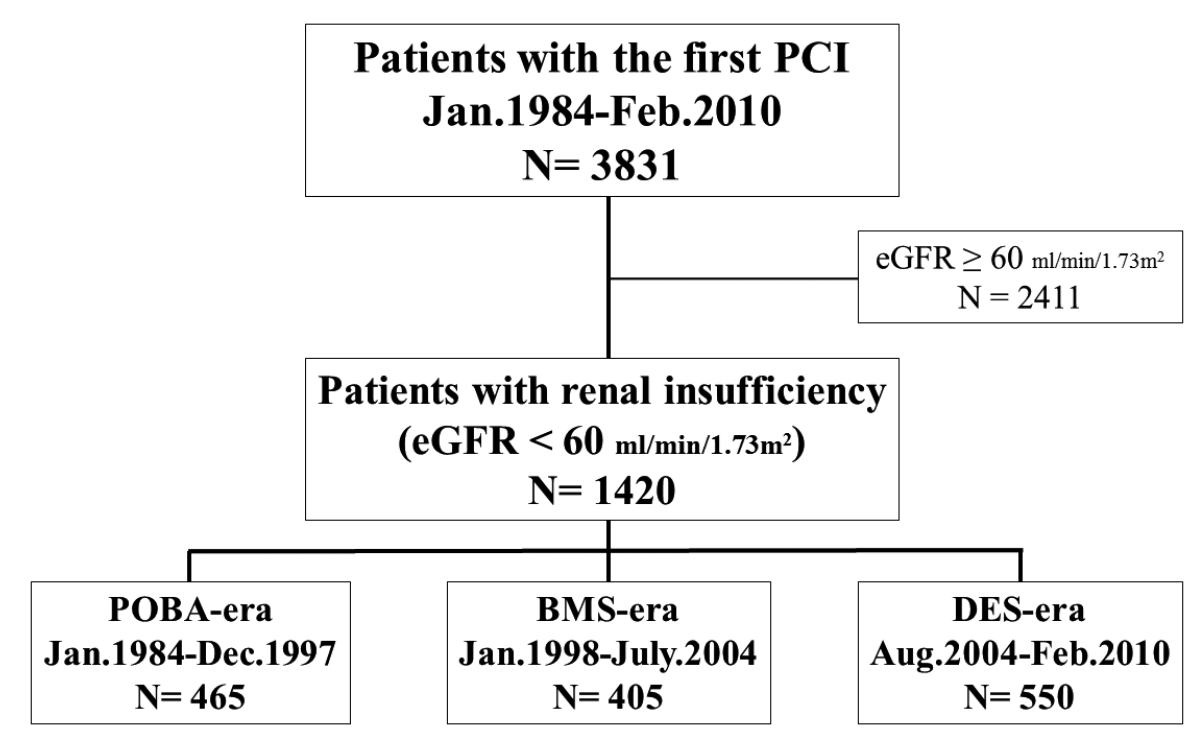

Fig. 1. A flow chart of the study population

A total of 1,420 patients with renal insufficiency who underwent the first percutaneous coronary intervention in our institution were analyzed in this study.

$P$-value $<0.05$ was considered to be statistically significant. All data were analyzed using JMP version 10.0 for Windows (SAS Institute, Cary, NC).

\section{Results}

A flow chart of the study population is provided in Fig. 1. A total of 1,420 patients were analyzed (POBA era, $n=465$; BMS era, $n=405$; and DES era, $n=550)$. The baseline characteristics are shown in Table 1. The mean age was highest in the DES era and the prevalence of hypertension and diabetes. The percentage of severe renal insufficiency $(e G F R<30)$ was higher, and the prevalence of hemodialysis was also higher in the DES era. The percentage of current smoking was higher in the POBA era than in the other eras. Lipid profiles were more favorable in the DES and BMS eras than in the POBA era. HbAlc was higher in the POBA era, whereas hemoglobin level and left ventricular ejection fraction were lower in the DES and BMS eras. The success rate of PCI was also higher in these eras than in the POBA era. In addition, the DES and BMS eras included more patients who presented with ACS at the time of the initial PCI. The use of evidence-based medical therapy (i.e., aspirin, statins, $\mathrm{ACEI} / \mathrm{ARB}$, and $\beta$-blockers) for secondary prevention of cardiovascular events significantly increased over time (Table 1). Lesion characteristics showed that diseased vessels differed between the groups, and a higher prevalence of MVD was noted in the DES and BMS eras than in the POBA era (Table
1). Information regarding 3-year clinical outcomes was collected from all patients in this study. The incidence of each individual clinical outcome is provided in Table 2. The unadjusted cumulative event-free survival rate for 3-year MACE was significantly different across the three eras (Fig. 2). The incidence of 3-year MACE was reduced over the three eras ( $P$ for trend $=0.0009)$ (Fig. 3). The univariate Cox regression analysis for 3-year MACE showed that the relative risk reduction was 23\% [HR: $0.77,95 \%$ confidential interval $(\mathrm{CI}): 0.61-0.96, P=0.02]$ in the BMS era and 35\% (HR: $0.65,95 \%$ CI: $0.52-0.80, P<0.0001$ ) in the DES era (using the POBA era as reference). Table 3 shows that adjusted relative risk reduction for 3-year MACE was 35\% (HR: 0.65, 95\% CI: 0.51-0.84, $P=0.0009$ ) in the BMS era and 51\% (HR: 0.49, 95\% CI: $0.38-0.63, P<0.0001)$ in the DES era. Compared with the BMS era, the adjusted relative risk reduction of the DES era was 26\% (HR: $0.74,95 \%$ CI: $0.58-0.95, P=0.02)$. High age, male gender, low BMI, diabetes mellitus, ACS, and hemodialysis were also associated with increased 3-year MACE (Table 3). Regarding all-cause mortality that is considered to be an important endpoint for patients with renal insufficiency, multivariable Cox regression analysis revealed that the DES era had a tendency toward lower incidence of 3-year all-cause mortality compared with the POBA era (Fig.4). 
Table 1. Patients' characteristics

\begin{tabular}{|c|c|c|c|c|}
\hline & $\begin{array}{l}\text { POBA era } \\
(N=465)\end{array}$ & $\begin{array}{l}\text { BMS era } \\
(N=405)\end{array}$ & $\begin{array}{l}\text { DES era } \\
(N=550)\end{array}$ & $p$ value \\
\hline Age, year & $61.6 \pm 9.6$ & $67.3 \pm 9.0^{*}$ & $69.8 \pm 9.9^{*}$ & $<0.0001$ \\
\hline Male, $n(\%)$ & $395(85.0)$ & $324(80.0)$ & $440(80.0)$ & 0.07 \\
\hline BMI, $\mathrm{kg} / \mathrm{m}^{2}$ & $23.5 \pm 3.7$ & $24.0 \pm 3.8$ & $23.8 \pm 3.3$ & 0.2 \\
\hline Hypertension, $n(\%)$ & $310(66.7)$ & $291(71.9)$ & $429(78.0)^{\dagger}$ & 0.000 \\
\hline Diabetes mellitus, $n(\%)$ & 167 (35.9) & $164(40.5)$ & $245(44.6)$ & 0.02 \\
\hline Dyslipidemia, $n(\%)$ & $348(74.1)$ & $252(62.5)$ & $390(70.9)$ & 0.001 \\
\hline Current smoking, $n(\%)$ & $273(59.1)$ & $74(18.4)^{\dagger}$ & $112(20.4)^{\dagger}$ & $<0.0001$ \\
\hline Family history, $n(\%)$ & $122(26.3)$ & $115(28.9)$ & $138(25.1)$ & 0.5 \\
\hline LDL-C, mg/dL & $133.8 \pm 42.9$ & $116.4 \pm 32.1^{*}$ & $109.8 \pm 33.3^{*}$ & $<0.0001$ \\
\hline HDL-C, mg/dL & $42.6 \pm 12.1$ & $44.2 \pm 13.7$ & $43.2 \pm 13.1$ & 0.2 \\
\hline $\mathrm{TC}, \mathrm{mg} / \mathrm{dL}$ & $205.3 \pm 47.8$ & $187.5 \pm 6.6^{*}$ & $180.6 \pm 9.0^{*}$ & $<0.0001$ \\
\hline $\mathrm{TG}, \mathrm{mg} / \mathrm{dL}$ & $144.4 \pm 73.4$ & $134.7 \pm 82.2$ & $135.3 \pm 75.9$ & 0.09 \\
\hline HbA1c (NGSP), \% & $7.0 \pm 3.2$ & $6.2 \pm 1.2^{*}$ & $6.2 \pm 1.1^{*}$ & 0.009 \\
\hline $\mathrm{Hb}, \mathrm{g} / \mathrm{dL}$ & $13.3 \pm 1.7$ & $12.8 \pm 2.0^{*}$ & $12.6 \pm 2.1^{*}$ & 0.000 \\
\hline LVEF, \% & $65.3 \pm 13.1$ & $63.4 \pm 14.0$ & $58.6 \pm 14.2^{*}$ & $<0.0001$ \\
\hline $\mathrm{eGFR}, \mathrm{mL} / \mathrm{min} / 1.73 \mathrm{~m}^{2}$ & $46.7 \pm 12.5$ & $45.2 \pm 14.8$ & $42.5 \pm 17.7^{*}$ & $<0.0001$ \\
\hline Severity of renal insufficiency & & & & $<0.0001$ \\
\hline eGFR $<15$ & $23(5.0)$ & $31(7.7)$ & $83(15.1)$ & \\
\hline $15 \leq \mathrm{eGFR}<30$ & $16(3.44)$ & $22(5.4)$ & $19(3.4)$ & \\
\hline $30 \leq \mathrm{eGFR}<45$ & $101(21.7)$ & $85(21.0)$ & $105(19.1)$ & \\
\hline $45 \leq \mathrm{eGFR}<60$ & $325(69.9)$ & $267(65.9)$ & $343(62.4)$ & \\
\hline Hemodialysis, $n(\%)$ & $18(3.9)$ & $31(7.7)^{\dagger}$ & $81(14.7)^{\dagger}$ & $<0.0001$ \\
\hline Acute coronary syndrome, $n(\%)$ & $56(12.0)$ & $93(23.0)^{\dagger}$ & $148(26.9)^{\dagger}$ & $<0.0001$ \\
\hline Success rate, $n(\%)$ & $401(86.2)$ & $385(95.1)^{\dagger}$ & $526(95.6)^{\dagger}$ & $<0.0001$ \\
\hline Type of procedure, $n(\%)$ & & & & $<0.0001$ \\
\hline POBA & $448(96.3)$ & $79(19.8)$ & $34(6.4)$ & \\
\hline BMS & $17(3.7)$ & $320(80.2)$ & $188(35.1)$ & \\
\hline DES & $0(0)$ & $0(0)$ & $313(58.5)$ & \\
\hline \multicolumn{5}{|l|}{ Medication, $n(\%)$} \\
\hline Aspirin & $324(70.7)$ & $354(90.3)^{\dagger}$ & $511(93.3)^{\dagger}$ & $<0.0001$ \\
\hline Other anti-platelet agent & $355(78.0)$ & $278(70.9)$ & $474(86.7)$ & $<0.0001$ \\
\hline Statin & $78(35.5)$ & $151(38.3)$ & $305(55.6)^{\dagger}$ & $<0.0001$ \\
\hline ACEI/ARB & $52(11.4)$ & $173(44.1)^{\dagger}$ & $303(55.3)^{\dagger}$ & $<0.0001$ \\
\hline$\beta$-blocker & $124(27.1)$ & $171(43.6)^{\dagger}$ & $286(52.2)^{\dagger}$ & $<0.0001$ \\
\hline Calcium channel blocker & $233(50.9)$ & $168(43.0)^{\dagger}$ & $236(43.1)^{\dagger}$ & 0.02 \\
\hline Diseased vessel & & & & $<0.0001$ \\
\hline LAD & $223(48.0)$ & $165(40.7)$ & $237(43.1)$ & \\
\hline LCX & $64(13.8)$ & $77(19.0)$ & $112(20.4)$ & \\
\hline RCA & $120(25.8)$ & $141(34.8)$ & $171(31.1)$ & \\
\hline LMT & $7(1.5)$ & $5(1.2)$ & $19(3.5)$ & \\
\hline Others & $51(11.0)$ & $17(4.2)$ & $11(2.0)$ & \\
\hline Number of diseased vessel & $1.6 \pm 0.8$ & $1.7 \pm 0.8$ & $1.9 \pm 0.8^{*}$ & $<0.0001$ \\
\hline MVD & $214(46.6)$ & $198(48.9)^{\dagger}$ & $357(65.4)^{\dagger}$ & $<0.0001$ \\
\hline \multicolumn{5}{|l|}{ QCA } \\
\hline Reference diameter, $\mathrm{mm}$ & $3.0 \pm 0.7$ & $3.0 \pm 0.5$ & $2.8 \pm 0.5^{*}$ & $<0.0001$ \\
\hline MLD (pre), mm & $0.59 \pm 0.41$ & $0.51 \pm 0.46$ & $0.42 \pm 0.37^{*}$ & 0.000 \\
\hline MLD (post), mm & $2.15 \pm 0.74$ & $2.57 \pm 0.77^{*}$ & $2.66 \pm 0.58^{*}$ & $<0.0001$ \\
\hline Stent diameter, $\mathrm{mm}$ & & $3.17 \pm 0.4$ & $2.97 \pm 0.41$ & $<0.0001$ \\
\hline Stent length, mm & & $16.7 \pm 4.2$ & $19.7 \pm 6.2$ & $<0.0001$ \\
\hline
\end{tabular}

BMI; body mass index, LDL-C; low density lipoprotein cholesterol, HDL-C; high density lipoprotein cholesterol, TC; total cholesterol, TG; triglyceride, HbA1c; glycocilated hemoglobin, Hb; hemoglobin, LVEF; left ventricular ejection fraction, eGFR; estimated glomerular filtration rate, POBA; plain old balloon angioplasty, BMS; bare metal stents, DES; drug-eluting stents, ACEI; angiotensin converting enzyme inhibitor, ARB; angiotensin receptor blocker, LAD; left anterior descending artery, LCX; left circumflex, RCA; right coronary artery, LMT; left main trunk, MVD; multivessel coronary artery disease, QCA; quantitative coronary angiography, MLD; minimum lumen diameter 
Table 2. The incidence rate of each individual clinical outcome

\begin{tabular}{lcll}
\hline & $\begin{array}{c}\text { POBA era } \\
(N=465)\end{array}$ & $\begin{array}{c}\text { BMS era } \\
(N=405)\end{array}$ & $\begin{array}{c}\text { DES era } \\
(N=550)\end{array}$ \\
\hline All-cause mortality, (\%) & $38(8.1)$ & $46(11.4)$ & $80(14.5)$ \\
Non-fatal acute coronary syndrome, (\%) & $14(3.0)$ & $28(6.9)$ & $26(4.7)$ \\
Non-fatal stroke, (\%) & $5(1.1)$ & $27(6.7)$ & $21(3.8)$ \\
Repeat revascularization, (\%) & $140(30.1)$ & $64(15.8)$ & $61(11.1)$ \\
\hline
\end{tabular}

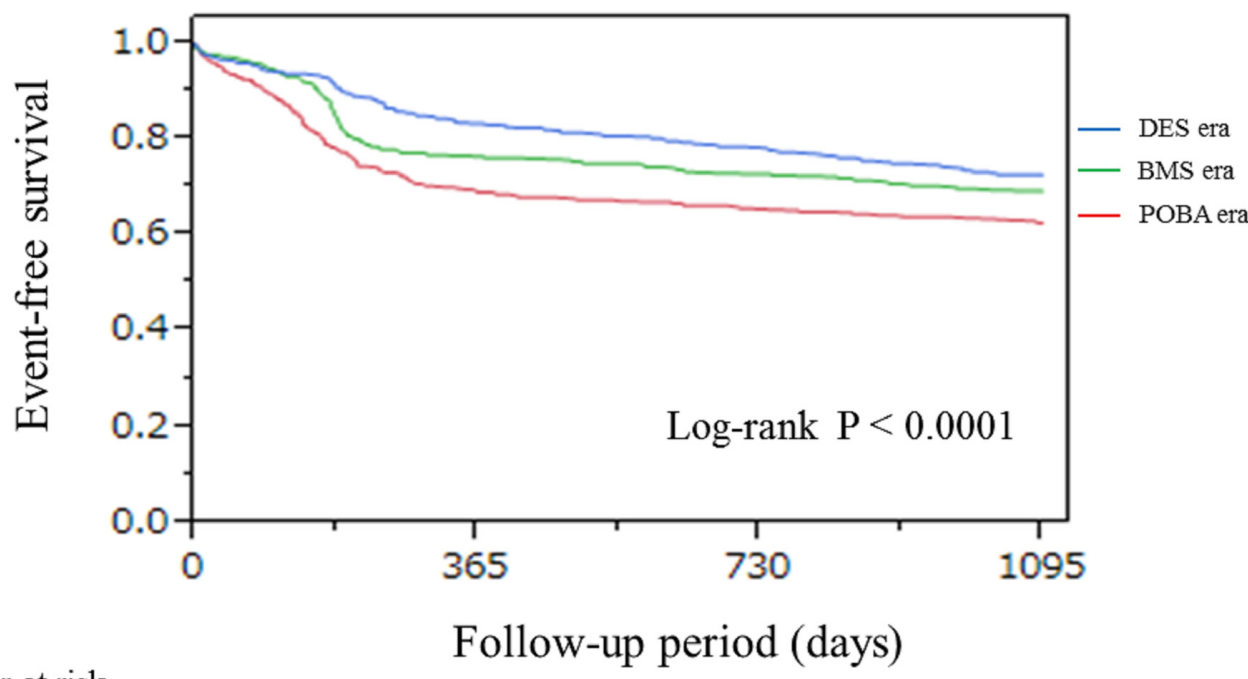

Number at risk

$\begin{array}{lllll}\text { DES era } & 550 & 456 & 428 & 397 \\ \text { BMS era } & 405 & 308 & 293 & 279 \\ \text { POBA era } & 465 & 321 & 304 & 291\end{array}$

Fig. 2. Kaplan-Meier curves for 3-year MACE

Kaplan-Meier curves for 3-year major adverse cardiovascular events show a significant difference across the three eras. The event rate was the lowest in the drug-eluting stent era.

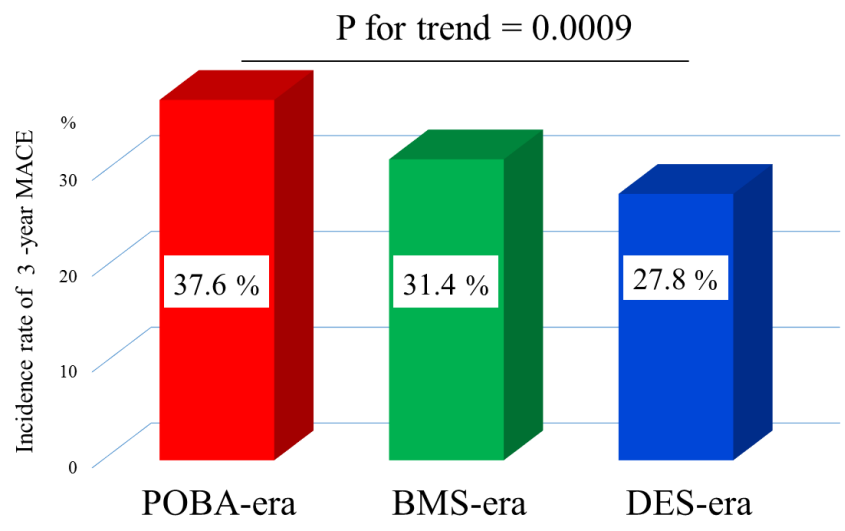

Fig. 3. The overall incidence of 3-year major adverse cardiovascular events across three eras

Unadjusted incidence of 3-year major adverse cardiovascular events was significantly decreased across plain old balloon angioplasty, bare metal stent, and drug-eluting stent eras.

\section{Discussion}

The main findings of this study that examined the clinical outcomes following PCI in patients with renal insufficiency are as follows: (1) the baseline characteristics have become unfavorable over the recent two decades in terms of higher age and higher prevalence of comorbid diseases (hypertension, diabetes, and hemodialysis); (2) the success rate of PCI and the use of evidence-based medical therapy for secondary prevention have increased over time; and (3) the incidence of 3-year MACE has significantly decreased over time, whereas the incidence of 3-year all-cause mortality tended to be lower in the DES era. These findings suggest that the improvement of PCI devices and the increased use of evidence-based medical therapy for secondary prevention of CAD have led to the improved long-term clinical outcomes following PCI 
Table 3. Multivariable Cox regression analyses for 3-year major adverse cardiovascular events

\begin{tabular}{lccc}
\hline & Hazard ratio & $95 \%$ CI & $p$ \\
\hline Age, 1 year increase & 1.02 & $1.01-1.03$ & 0.000 \\
Male gender, yes & 1.35 & $1.04-1.76$ & 0.02 \\
BMI, $1 \mathrm{~kg} / \mathrm{m}^{2}$ increase & 0.97 & $0.95-0.99$ & 0.049 \\
Diabetes mellitus, yes & 1.26 & $1.04-1.53$ & 0.017 \\
Dyslipidemia, yes & 0.99 & $0.81-1.22$ & 0.9 \\
Hypertension, yes & 1.08 & $0.87-1.34$ & 0.5 \\
Current smoking, yes & 1.08 & $0.86-1.34$ & 0.5 \\
ACS, yes & 1.45 & $1.15-1.81$ & 0.002 \\
Hemodialysis, yes & 2.03 & $1.51-2.69$ & $<0.0001$ \\
BMS era (POBA era as reference) & 0.65 & $0.51-0.84$ & 0.001 \\
DES era (POBA era as reference) & 0.49 & $0.38-0.63$ & $<0.0001$ \\
\hline
\end{tabular}

BMI; body mass index, ACS; acute coronary syndrome, BMS; bare metal stent, POBA; plain old balloon angioplasty, DES; drug-eluting stent

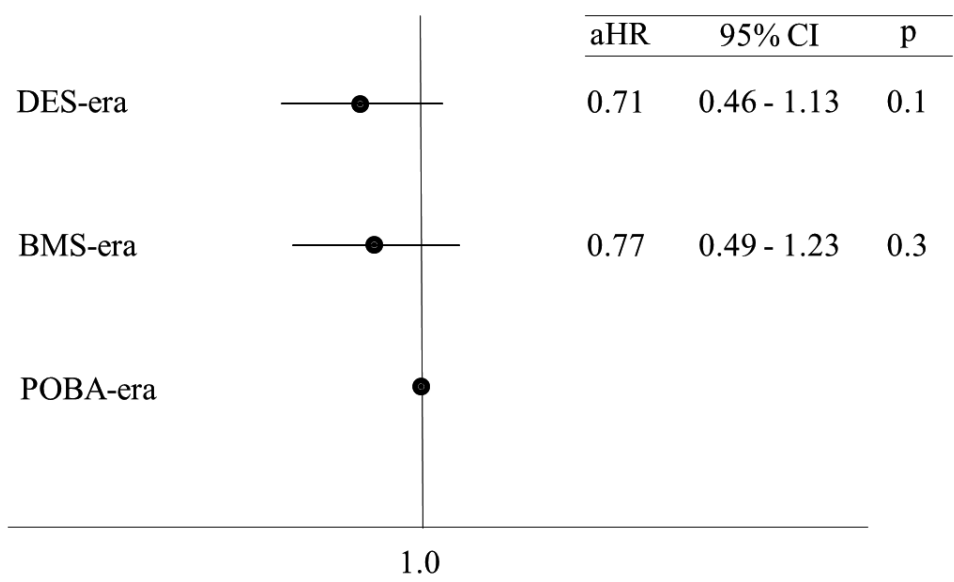

Fig. 4. Cox regression analysis for 3-year all-cause mortality

The forest plot demonstrates adjusted hazard ratio and $95 \%$ confidence interval for 3-year all-cause mortality of drug-eluting stent and bare metal stent eras against plain old balloon angioplasty era after controlling following variables (age, male gender, body mass index, diabetes mellitus, hypertension, dyslipidemia, current smoking, hemodialysis, and acute coronary syndrome).

mainly because of the reduction in repeat revascularization, even in high-risk patients with renal insufficiency. To the best of our knowledge, this is the first study examining clinical outcomes following PCI across the POBA, BMS, and DES eras in patients with renal insufficiency.

Renal insufficiency is considered to be an independent risk factor for $\mathrm{CAD}^{1-6)}$; furthermore, $\mathrm{CAD}$ patients with renal insufficiency have worse clinical outcomes compared with patients without renal insufficiency ${ }^{7-9)}$. Coronary revascularization has been demonstrated to be effective in improving prognosis in CAD patients with renal insufficiency ${ }^{18,19)}$. In addition, PCI has been evolving with new devices, improved operator skills, and the establishment of medical therapy for secondary prevention.

To date, there are few studies examining the temporal trends in clinical outcomes after PCI in patients with renal insufficiency. Krishnaswami et al. conducted a retrospective study evaluating the temporal trends in mortality following coronary revascularization in patients with end-stage renal disease requiring hemodialysis during the following three time periods: 1996-1999, 2000-2003, and 2004-2008 ${ }^{20)}$. They reported that the relative risk reduction for 3-year mortality after coronary revascularization was $34 \%$ in the 2004-2008 period compared with the 1996-1999 period. The result was partially consistent with our 
results that showed a significant reduction in 3-year MACE and a tendency toward reduction in 3-year allcause mortality over 26 years. However, in that study, not only PCI but also coronary artery bypass grafting was included as a coronary revascularization strategy, which differed from our study. Thus, no study has reported the temporal trends in clinical outcomes after PCI in patients with renal insufficiency using a longitudinal cohort. In this regard, our study is considered to be novel. In our study, the adjusted risk for 3-year all-cause mortality tended to be lower in the DES era than in the POBA era. The adjusted relative risk for 3-year MACE was the lowest in the DES era compared with the POBA and the BMS eras, and also the relative risk was lower in the BMS era than in the POBA era, despite the higher risk profiles of the patients in the later time periods. Reduction in the incidence of cardiovascular events over the 26-year period of this study could be explained by the following: advances in PCI devices such as the advent of DES, the high rate of PCI success, the increased use of evidence-based therapies for secondary prevention (i.e., aspirin, statins, $\beta$-blockers, and ACEI/ARBs), and smoking cessation. Indeed, the Global Registry of Acute Coronary Events has previously shown that adherence to guideline-recommended medical therapy for secondary prevention yielded favorable clinical outcomes after acute coronary events ${ }^{21)}$. The present study could help us ascertain the importance of advances in PCI technology, medical therapy for secondary prevention, and smoking cessation in reducing cardiovascular events, even in high-risk patients with renal insufficiency following PCI.

\section{Limitations}

Our study has several limitations. First, this study was conducted at a single institution, and the study population was small, limiting the generalizability of our results. However, our data are important because PCI has been utilized in our institution from the beginning of the use of PCI in Japan. Second, there was a lack of data regarding medication use, causes of renal insufficiency, and detailed information about the volume of contrast medium used in the index PCI and the effect on periprocedural deterioration of renal function. Third, because of the long-term follow-up period across three generations of PCI, unmeasured factors that were not assessed in this study might have effects on the incidence of clinical outcomes.

\section{Future Perspectives}

The present study and the previous reports indicate the importance of adhering to evidence-based medical and nonpharmacological therapy for secondary prevention and the necessity of further establishing renewed measures to reduce fatal cardiovascular events. Considering that increased physical activity and nutrition therapy have been demonstrated to have positive effects on lowering cardiovascular events ${ }^{22,23)}$, future studies are necessary to consider nonpharmacological interventions for such issues for the prevention of cardiovascular events.

\section{Conclusion}

Long-term clinical outcomes following PCI in patients with renal insufficiency in our institution improved over the past 26 years mainly because of the reduction in repeat revascularization, despite the higher risk profiles of the patients in the recent era.

\section{Acknowledgments}

This study was supported by a grant for scientific research from the Ministry of Health, Labor and Welfare (23591063). We gratefully acknowledge the contributions made by Ms. Yumi Nozawa and Ms. Ayako Onodera in data collection.

\section{Disclosures}

Dr Daida has received Speakers' Bureau Honoraria from MSD K.K., AstraZeneca K.K., Kowa Pharmaceutical Company LTD., Sanofi-Aventis K.K., GlaxoSmithKline K.K., Shionogi \& Co., Ltd., Daiichi-Sankyo Company, Limited, Takeda Pharmaceutical Co., Ltd., Mitsubishi Tanabe Pharma Corp., Pfizer Co., Ltd., and Astellas Pharma Inc. and research funds from Takeda Pharmaceutical Co., Ltd., Bristol-Myers Squibb Company, Nippon Boehringer Ingelheim Co., Ltd., Astellas Pharma Inc., Novartis Pharma K.K., MSD K.K., Sanofi-Aventis K.K., Otsuka Pharmaceutical Co., Ltd., Dainippon Sumitomo Pharma Co., Ltd., Pfizer Co., Ltd., Kowa Pharmaceutical Company LTD., Shionogi \& Co., Ltd., AstraZeneca K.K., Teijin Limited, and Morinaga Milk Industry Co., Ltd..

Dr Miyauchi has received speakers' Bureau Honoraria from MSD K.K., AstraZeneca K.K., Kowa Pharmaceutical Company LTD., Sanofi-Aventis K.K., Shionogi \& Co., Ltd., Daiichi-Sankyo Company, Limited, Takeda Pharmaceutical Co., Ltd., Pfizer Co., Ltd., Astellas Pharma Inc., and Novartis Pharma K.K.

The other authors report no conflicts. 


\section{References}

1) Anavekar NS, McMurray JJ, Velazquez EJ, Solomon SD, Kober L, Rouleau JL, White HD, Nordlander R, Maggioni A, Dickstein K, Zelenkofske S, Leimberger JD, Califf RM, Pfeffer MA: Relation between renal dysfunction and cardiovascular outcomes after myocardial infarction. N Engl J Med, 2004; 351: 1285-1295

2) McCullough PA, Jurkovitz CT, Pergola PE, McGill JB, Brown WW, Collins AJ, Chen SC, Li S, Singh A, Norris KC, Klag MJ, Bakris GL; KEEP Investigators: Independent components of chronic kidney disease as a cardiovascular risk state: results from the Kidney Early Evaluation Program (KEEP). Arch Intern Med, 2007; 167: 11221129

3) Athyros VG, Katsiki N, Karagiannis A, Mikhailidis DP: should chronic kidney disease be considered as a coronary heart disease equivalent? Curr Vasc Pharmacol, 2012; 10: 374-377

4) Polonsky TS, Bakris GL: Chronic kidney disease: a coronary heart disease equivalent? Lancet, 2012; 380: 783785

5) McClellan WM, Newsome BB, McClure LA, Cushman M, Howard G, Audhya P, Abramson JL, Warnock DG: Chronic kidney disease is often unrecognized among patients with coronary heart disease: the REGARDS Cohort Study. Am J Nephrol, 2009; 29: 10-17

6) Chen YL, Chen CH, Wallace CG, Wang HT, Yang CC, Yip HK: Levels of circulating microparticles in patients with chronic cardiorenal disease. J Atheroscler Thromb, 2015; 22: 247-256

7) Yamaguchi J, Kasanuki $H$, Ishii $Y$, Yagi M, Ogawa $H$, Fujii SY, Koganei H, Okada H, Kimura H, Horie T, Haze K, Sumiyoshi T, Honda T; HIJC Study Group: Prognostic significance of serum creatinine concentration for inhospital mortality in patients with acute myocardial infarction who underwent successful primary percutaneous coronary intervention (from the Heart Institute of Japan acute myocardial infarction [HIJAMI] registry). Am J Cardiol, 2004; 93: 1526-1528

8) Yagi $H$, Kawai $M$, Komukai $K$, Ogawa $T$, Minai $K$, Nagoshi T, Ogawa K, Sekiyama H, Taniguchi I, Yoshimura M: Impact of chronic kidney disease on the severity of initially diagnosed coronary artery disease and the patient prognosis in the Japanese population. Heart Vessels, 2011; 26: 370-378

9) Kanda D, Takumi T, Miyata M, Tokushige A, Sonoda T, Yoshino S, Saihara K, Ohishi M: Angiotensin-Converting Enzyme Inhibitor Prevents the Worsening of Renal Function in the Late Phase after Percutaneous Coronary Intervention. J Atheroscler Thromb, 2016; 23: 233-240

10) Grüntzig AR, Senning A, Siegenthaler WE: Nonoperative dilatation of coronary-artery stenosis: percutaneous transluminal coronary angioplasty. N Engl J Med, 1979; 301: 61-68

11) Stone GW, Ellis SG, Cox DA, Hermiller J, O'Shaughnessy C, Mann JT, Turco M, Caputo R, Bergin P, Greenberg J, Popma JJ, Russell ME; TAXUS-IV Investigators: A polymer-based, paclitaxel-eluting stent in patients with coronary artery disease. N Engl J Med, 2004; 350: 221-231

12) Stone GW, Rizvi A, Newman W, Mastali K, Wang JC,
Caputo R, Doostzadeh J, Cao S, Simonton CA, Sudhir K, Lansky AJ, Cutlip DE, Kereiakes DJ; SPIRIT IV Investigators: Everolimus-eluting versus paclitaxel-eluting stents in coronary artery disease. N Engl J Med, 2010; 362: 1663-1674

13) Moses JW, Leon MB, Popma JJ, Fitzgerald PJ, Holmes DR, O'Shaughnessy C, Caputo RP, Kereiakes DJ, Williams DO, Teirstein PS, Jaeger JL, Kuntz RE; SIRIUS Investigators: Sirolimus-eluting stents versus standard stents in patients with stenosis in a native coronary artery. N Engl J Med, 2003; 349: 1315-1323

14) Morice MC, Serruys PW, Sousa JE, Fajadet J, Ban Hayashi E, Perin M, Colombo A, Schuler G, Barragan P, Guagliumi G, Molnàr F, Falotico R; RAVEL Study Group: Randomized Study with the Sirolimus-Coated Bx Velocity Balloon-Expandable Stent in the Treatment of Patients with de Novo Native Coronary Artery Lesions. A randomized comparison of a sirolimus-eluting stent with a standard stent for coronary revascularization. N Engl J Med, 2002; 346: 1773-1780

15) Naito R, Miyauchi K, Konishi H, Tsuboi S, Ogita M, Dohi T, Kasai T, Tamura H, Okazaki S, Isoda K, Daida H: Temporal Trends in Clinical Outcome After Percutaneous Coronary Intervention 1984-2010 - Report From the Juntendo PCI Registry. Circ J, 2015 Oct 28. [Epub ahead of print]

16) Matsuo S, Imai E, Horio M, Yasuda $Y$, Tomita K, Nitta K, Yamagata K, Tomino Y, Yokoyama H, Hishida A; Collaborators developing the Japanese equation for estimated GFR: Collaborators developing the Japanese equation for estimated GFR: Revised equations for estimated GFR from serum creatinine in Japan. Am J Kidney Dis, 2009; 53: 982-992

17) Kashiwagi A, Kasuga M, Araki E, Oka Y, Hanafusa T, Ito $\mathrm{H}$, Tominaga $\mathrm{M}$, Oikawa S, Noda M, Kawamura T, Sanke T, Namba M, Hashiramoto M, Sasahara T, Nishio Y, Kuwa K, Ueki K, Takei I, Umemoto M, Murakami M, Yamakado M, Yatomi Y, Ohashi H; Committee on the Standardization of Diabetes Mellitus - Related Laboratory Testing of Japan Diabetes Society: International clinical harmonization of glycated hemoglobin in Japan: from Japan Diabetes Society to National Glycohemoglobin Standardization Program values. J Diabetes Investig, 2012; 3: 39-40

18) Reddan DN, Szczech LA, Tuttle RH, Shaw LK, Jones RH, Schwab SJ, Smith MS, Califf RM, Mark DB, Owen WF Jr: Chronic kidney disease, mortality, and treatment strategies among patients with clinically significant coronary artery disease. J Am Soc Nephrol, 2003; 14: 23732380

19) Hemmelgarn BR, Southern D, Culleton BF, Mitchell LB, Knudtson ML, Ghali WA; Alberta Provincial Project for Outcomes Assessment in Coronary Heart Disease (APPROACH) Investigators: Survival after coronary revascularization among patients with kidney disease. Circulation, 2004; 110: 1890-1895

20) Krishnaswami A, Leong TK, Hlatky MA, Chang TI, Go AS: Temporal trends in mortality after coronary artery revascularization in patients with end-stage renal disease. Perm J, 2014; 18: 11-16

21) Fox KA, Steg PG, Eagle KA, Goodman SG, Anderson FA 
Jr, Granger CB, Flather MD, Budaj A, Quill A, Gore JM; GRACE Investigators: Decline in rates of death and heart failure in acute coronary syndromes, 1999-2006. JAMA, 2007; 297: 1892-1900

22) Booth JN 3rd, Levitan EB, Brown TM, Farkouh ME, Safford MM, Muntner P: Effect of sustaining lifestyle modifications (nonsmoking, weight reduction, physical activity, and mediterranean diet) after healing of myocardial infarction, percutaneous intervention, or coronary bypass (from the REasons for Geographic and Racial Differences in Stroke Study). Am J Cardiol, 2014; 113: 1933-1940

23) Knoops KT, de Groot LC, Kromhout D, Perrin AE, Moreiras-Varela O, Menotti A, van Staveren WA: Mediterranean diet, lifestyle factors, and 10-year mortality in elderly European men and women: the HALE project. JAMA, 2004; 292: 1433-1439 